\title{
An explicit estimation of $p$-adic sizes of common zeros of partial derivative polynomials associated with a complete quartic form
}

\begin{abstract}
In this paper we obtain an estimation of p-adic sizes of common zeros of partial derivative polynomials associated with a complete quartic polynomial by applying the Newton polyhedron technique. Such estimates are obtained by examining indicator diagrams associated with the Newton polyhedra of partial derivatives polynomials considered and applying new conditions to improve the results of earlier researchers.
\end{abstract}

Keyword: Indicator diagram; Newton polyhedron; P-sizes 